\title{
EL GÉNERO OROBANCHE L. (OROBANCHACEAE) EN LA PROVINCIA DE ALMERÍA, SE DE ESPAÑA
}

\author{
Antonio PUJADAS SALVÁ y Angel LORA GONZÁLEZ
}

\begin{abstract}
RESUMEN. El género Orobanche L. (Orobanchaceae) en la provincia de Almería, SE de España. Se ha llevado a cabo la revisión del género Orobanche en la provincia de Almería (España). Para ello se ha realizado una exhaustiva prospección botánica y una revisión crítica de los principales herbarios nacionales. Para las dieciséis especies identificadas aportamos su corología, características ecológicas y hospedantes. Resaltamos la presencia de dos nuevas citas provinciales: O. schultzii Mutel y $O$. foetida Poir. subsp. broteri Guimarães.
\end{abstract}

Palabras clave. Orobanchaceae, Orobanche, jopo, parásito, corología, Almería, SE España.

ABSTRACT. The genus Orobanche (Orobanchaceae) in the province of Almería, SE of Spain. A botanical survey has been carried out in order to determine the Orobanche species ocurring in Almería (Spain). A revision of the main national herbaria has also been made. The geographical distribution, ecological characterization and hosts, for the sixteen Orobanche species are pointed out. Two new references are given: $O$. schultzii Mutel and $O$. foetida Poir. subsp. broteri Guimarães.

Key words. Orobanchaceae, Orobanche, broomrape, parasite, chorology, Almería, SE Spain.

\section{INTRODUCCIÓN}

Aunque son considerables los botánicos que han visitado la provincia de Almería y estudiado su flora ( $c f$. Sagredo, 1987; Castro y Cañadas, 1982; Fernández et al., 1991) es notable la ausencia casi total de estudios botánicos generales sobre dicha flora, siendo el primer tratado global la obra de Sagredo (1987).

Sobre Orobanche hay que resaltar que no existe ningún trabajo sobre el género para España, aunque sí existe para Portugal
(Guimarães, 1903). Una primera aproximación para la comunidad de Andalucía la llevamos a cabo en Pujadas Salvá et al. (1994). Para la provincia de Almería apenas aparecen referencias bibliográficas sobre el género antes de la flora de Sagredo (1987); en ella podemos encontrar el primer compendio sobre este grupo para la zona pero, aunque cita 20 especies de Orobanche, no llega a profundizar en su tratamiento, limitándose a descripciones muy generales y, junto a recolecciones propias, a citar referencias tomadas de otros autores que suponemos se hicieron sin ningún tipo de

Este trabajo ha sido financiado parcialmente gracias al proyecto de investigación PB93-1211 de la DGICYT. 
verificación y a veces usando sinónimos que pueden llevar a confusión.

Así entre las especies citadas por Sagredo (1987) no hemos podido contrastar la existencia de $O$. caesia Rchb., O. clausonis Pomel, $O$. coerulescens Stephan, O. purpurea Jacq., $O$. rapum-genistae Thuill., O. reticulata Wallr. y O. variegata Wallr. En Fernández et al. (1991) se recoge la presencia de casi todas éstas y además la de $O$. lavandulacea Rchb. De todas ellas se puede resaltar como muy poco probable la existencia de $O$. caesia, $O$. coerulescens y $O$. reticulata al ser especies de distribución eurosiberiana, no encontrándose en la Península Ibérica según Beck Von Mannagetta (1930). o. purpurea es de distribución centroeuropea y aunque se localiza en el NE peninsular su presencia en nuestra región es muy dudosa. Sobre O. clausonis (occidental-mediterránea), $O$. lavandulacea (esteno-mediterránea), $O$. rapum-genistae (europea-occidental) y $O$. variegata (de distribución tirrénica) cabría señalar como posible su existencia en el territorio aunque ésta no ha podido ser confirmada por ahora.

Dadas las dificultades y los problemas que se plantean en la identificación de estas especies, aportamos las descripciones morfológicas (breves) de las 16 especies de Orobanche localizadas en la provincia, además de unas claves de determinación tanto para material fresco como para material de herbario. Se indican también para cada una de ellas, las citas bibliográficas correspondientes a icones de calidad publicados en obras fácilmente accesibles. Aportamos tres icones originales correspondientes a $O$. schultzii Mutel, $O$. haenseleri Reut. y O. foetida Poir. subsp. broteri Guimarães ya que los que conocemos sobre estos taxones son incompletos o de difícil localización.

Se relacionan además las referencias correspondientes al material estudiado y los posibles hospedantes conocidos para cada una de las especies.

\section{MATERIAL Y MÉTODOS}

Desde 1986 estamos realizando el estudio florístico del género Orobanche en Andalucía, concentrándonos en principio en la provincia de Almería. Para llevar a cabo nuestro trabajo se ha efectuado una revisión bibliográfica basándonos principalmente en Willkomm (1870; 1893), Beck Von Mannagetta (1890; 1930), Chater \& Webb (1972) y Sagredo (1987). Igualmente se ha realizado una revisión crítica de los especímenes conservados en los principales herbarios regionales y nacionales: ALME, BC, BCC, BCF, COA, COLEGIO LA SALLE DE ALMERÍA, GDA, GDAC, JACA, JAEN, MA, MAF, MGC, MUB y SEV.

Además se ha llevado a cabo una prospección botánica en toda la provincia para la recolección de material vivo a fin de lograr una mayor precisión en la identificación y descripción de las especies, así como para la determinación de sus correspondientes hospedantes. Los testimonios de estas recolecciones se conservan en el Herbario COA.

Para la identificación y nomenclatura de los diferentes taxones hemos seguido principalmente a Beck Von Mannagetta (1890; 1930), Chater \& Webb (1972) y Greuter et al. (1989) para el género Orobanche, y a Tutin et al. (1964-1980) y Valdés et al. (1986) para las especies hospedantes.

\section{RESULTADOS}

Se han identificado dieciséis especies diferentes de Orobanche, cuya relación, descripción (sobre material almeriense), huéspedes y citas de herbario se comentan a continuación. Destacamos dos nuevas referencias para la provincia, O. schultzii y 0 . foetida subsp. broteri, así como la presencia de O. almeriensis recientemente descrita como nueva especie.

La mayoría de las especies aparecen en 

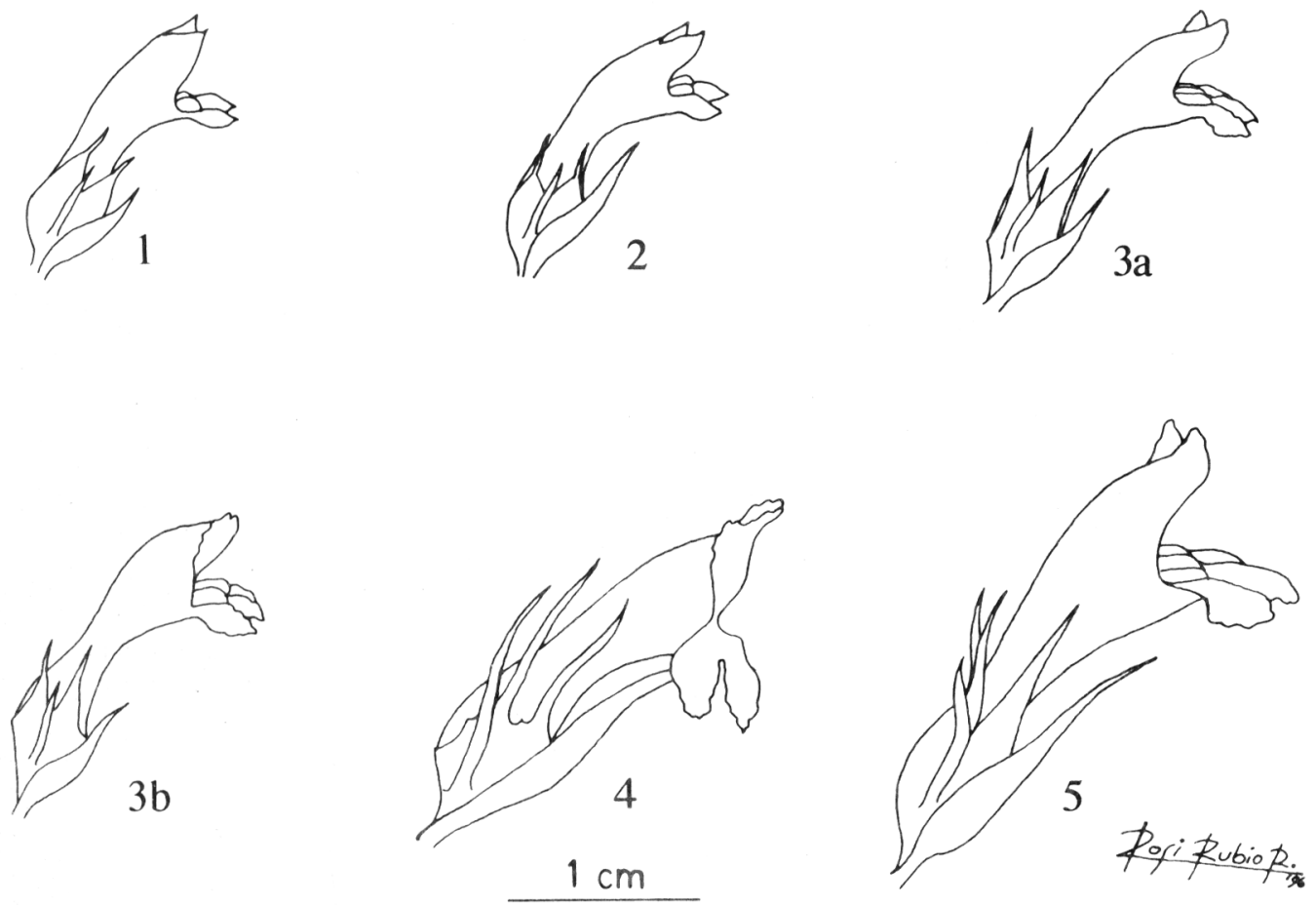

Figura l. Sect. Trionychon, aspectos morfológicos usados en las claves de determinación. Sect. Trionychon, morphological aspects used in the identification keys

ecosistemas ruderalizados o subruderalizados y frecuentemente sobre hospedantes característicos del matorral degradado heliófilo, a excepción de $O$. haenseleri que aparece sobre herbazales con un marcado carácter umbrófilo. La mayor parte presentan una distribución correspondiente a un amplio gradiente altitudinal, desde casi el nivel del mar hasta los $1500 \mathrm{msnm}$; este es el caso de $O$. ramosa, O. nana, O. mutelii, O. cernua, $O$. crenata, $O$. amethystea, $O$. minor, $O$. latisquama y $O$. gracilis var. spruneri. Algunas se han recolectado en zonas de montaña, a alturas comprendidas entre los 800 y 1000 msnm, como O. schultzii, O. almeriensis, $O$. artemisiae-campestris y $O$. foetida subsp. broteri. Por último, otras especies son características de zonas más templadas con temperaturas inferiores al resto de la provincia apareciendo por encima de los $1500 \mathrm{msnm}$, como ocurre con $O$. laevis (también a alturas algo inferiores), O. alba, O. haenseleri y $O$. gracilis var. gracilis.

\section{Clave para material fresco}

1. Inflorescencia con dos bractéolas y una bráctea por flor (fig. 1: 1-5)

1'. Inflorescencia con una bráctea por flor, sin bractéolas (fig. 2: 6-16) ... 6

2. Corola de (18)21-30 mm, campanuladoinfundibuliforme, azul violeta intenso, blanquecina en la base .................... 5. O. laevis

2'. Corola de 12-21 mm, de tubularinfundibuliforme a estrechamente campanulada, blanquecina en la base, blanco-amarillenta o azul en el ápice

\section{3}

3. Cáliz de (12)14-16 mm, con dientes 1,5-2 veces la longitud del tubo (fig. 1:4). 4. O. schultzii 3'. Cáliz de 4-10 mm con dientes igualando la longitud del tubo (fig. 1: 1-3 y 5) .................... 4 
4. Tallo de $8-12 \mathrm{~cm}$. Inflorescencia pauciflora. Dientes del cáliz triangulares con ápice acuminado-filiforme (fig. 1: 2). Corola con lóbulos del labio inferior agudos, azul brillante en el ápice. Anteras glabras

2. O. nana

4'. Tallo de (7)12-31 cm. Inflorescencias multifloras. Dientes del cáliz subulados (fig. 1: 3a) o acuminados (fig. 1:3b). Corola con lóbulos del labio inferior obtusos, blanquecina o azul en el ápice. Anteras glabras o pelosas .......... 5

5. Tallo de 17-31 cm, ramificado. Cáliz de 4-6 $\mathrm{mm}$. Corola de 12-15(17) $\mathrm{mm}$, blanquecina o azul pálido en el ápice. Parásito de plantas cultivadas

1. O. ramosa

5'. Tallo de (7)12-17(20) cm, usualmente simple, ocasionalmente ramificado. Cáliz de 7-10 mm. Corola de (15) 17-20 mm, azul brillante o pálido. Parásito de plantas silvestres .......3. O. mutélii

6. Estigma púrpura en la antesis .......................... 7

6'. Estigma blanco, rosado, amarillo o naranja en la antesis 10

7. Labio inferior de la corola glandular ciliado, numerosos pelos de la corola oscuros al menos en la base o en el ápice 7. O. alba

7'. Labio inferior de la corola no ciliado o subglabro, pelos de la corola incoloros o amarillo pálido

8. Segmentos del cáliz connatos en c. $1 / 2$ de su longitud (fig. 2: 11) .......... 11. O. almeriensis

8'. Segmentos del cáliz libres (fig. 2: 10 y 12). 9

9. Segmentos del cáliz desigualmente bífidos (fig. 2: 10) o enteros. Corola a menudo geniculada cerca de la base, pelosa glandular, blanquecina o blanco-amarillenta teñida de violeta o marrón en el ápice..... 10. $O$. amethystea

9'. Segmentos del cáliz desigualmente bidentados (fig. 2: 12). Corola no geniculada cerca de la base, puberulenta glandular o subglabra, blanquecina o amarillenta.

12. $O$. artemisiae-campestris

10. Estigma blanco 11

$10^{\prime}$. Estigma amarillo, naranja o rosado ....... 13

11. Segmentos del cáliz ampliamente connatos anterior y posteriormente. Corola de $25-30 \mathrm{~mm}$, blanco-amarillenta teñida de púrpura en los labios. Filamentos estaminales insertos a 8-12 $\mathrm{mm}$ de la base de la corola 14. O. latisquama

11'.Segmentos del cáliz libres o connatos en la base. Corola de (10)13-18 mm, blanca o azul- violeta oscuro en la mitad superior. Filamentos estaminales insertos a 2-6(8) $\mathrm{mm}$ de la base de la corola 12

12. Corola hinchada en la base, constricta e inflexa cerca de la mitad (fig. 2: 6), azul-violeta oscuro en su mitad superior. Filamentos insertos a (3) 4-6(8) $\mathrm{mm}$ de la base de la corola 6. O. cernua

12'. Corola tubular, débilmente curvada, no hinchada en la base ni constricta cerca de la mitad (fig. 2: 13), blanquecina. Filamentos insertos a 2-3 $\mathrm{mm}$ de la base de la corola ........

13. O. minor

13. Labio inferior de la corola no ciliado ......... 14

13'. Labio inferior de la corola ciliado .............. 15

14. Corola de 18-28 mm, con labios grandes y fuertemente divergentes (fig. 2: 9), pubescente glandular, blanca frecuentemente con venas lilas. Filamentos insertos a 2-3(4) $\mathrm{mm}$ de la base de la corola. Estigma amarillento, naranja o rosado 9. O. crenata

14'. Corola de (11)13-16 mm con labios cortos, subglabra, rojo-púrpura oscuro. Filamentos insertos a 1-2 $\mathrm{mm}$ de la base de la corola Estigma amarillo

16. O. foetida subsp. broteri

15. Corola rojo oscuro brillante interiormente...... 15a. O. gracilis var. gracilis

$15^{\prime}$. Corola no rojo oscuro interiormente ........... 16

16. Corola de (16) $18-24 \mathrm{~mm}$, de amarilla a ocre con venas rojas o marrones. Filamentos insertos a $1-2(2,5) \mathrm{mm}$ de la base de la corola

15b. $O$. gracilis var. spruneri

16'. Corola 20-25 mm, rojiza-naranja. Filamentos insertos a (2) 4-5 mm de la base de la corola ..

8. $O$. haenseleri

\section{Clave para pliegos de herbario}

1. Inflorescencia con dos bractéolas y una bráctea por flor (fig. 1: 1-5) ................................... 2

1'. Inflorescencia con una bráctea por flor, sin bractéolas (fig. 2: 6-16) .................................... 6

2. Dientes del cáliz 1,5-2 veces la longitud del tubo, lanceolados con ápice largamente filiforme (fig. 1: 4) .................... 4. O. schultzii

2'. Dientes del cáliz igualando la longitud del tubo; ápice no largamente filiforme (fig. 1: 1-3 y 5) ................ 3 


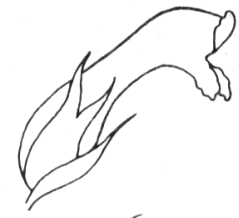

6

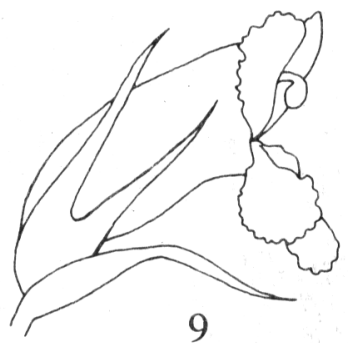

9

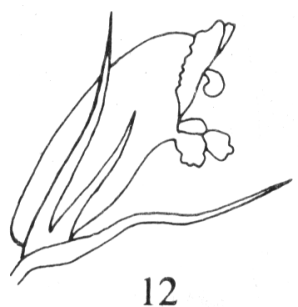

12

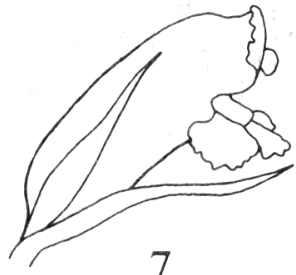

7

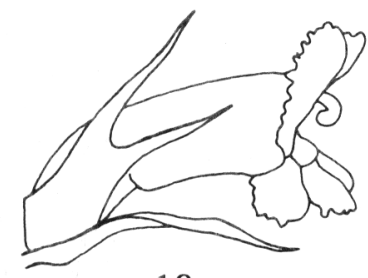

10

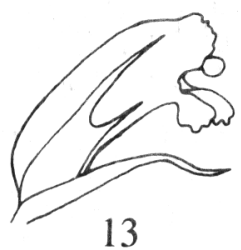

13
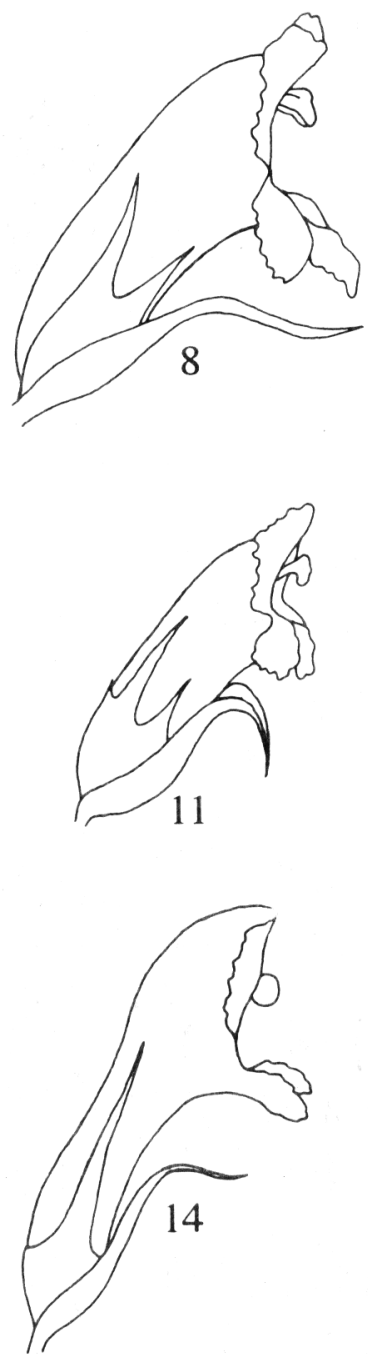

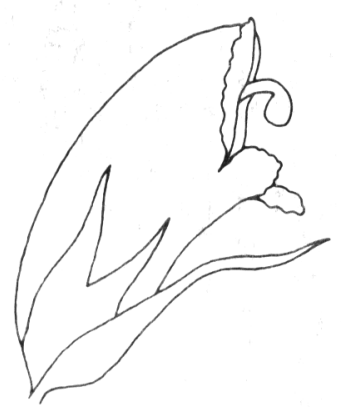

15

$1 \mathrm{~cm}$
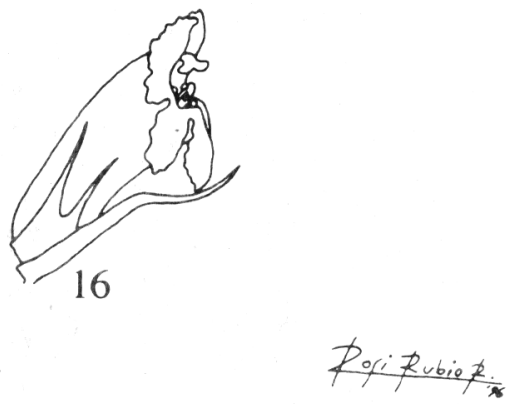

Figura 2. Sect. Orobanche, aspectos morfológicos usados en las claves de determinación. Sect. Orobanche, morphological aspects used in the identification keys. 
3. Cáliz de 13-18(21) mm. Corola de (18)21-30 $\mathrm{mm}$, campanulado-infundibuliforme

5. O. laevis

3'. Cáliz de 4-10 mm. Corola de 12-20 mm, tubularinfundibuliforme

4. Tallo de $8-12 \mathrm{~cm}$. Inflorescencia pauciflora. Dientes del cáliz triangulares con ápices acuminados filiformes (fig. 1: 2). Corola con lóbulos del labio inferior agudos. Anteras glabras ............................................ 2. O. nana

4'. Tallo de (7) $12-31 \mathrm{~cm}$. Inflorescencia multiflora. Dientes del cáliz subulados (fig. 1: 3a) o acuminados (fig. 1: 3b), no filiformes. Corola con lóbulos del labio inferior obtusos. Anteras glabras o pelosas

5

5. Tallo de 17-31 cm, ramificado. Cáliz de 4-6 mm. Corola de 12-15(17) mm .... 1. O ramosa

5'. Tallo de (7)12-17 cm, usualmente simple, ocasionalmente ramificado. Cáliz de $7-10 \mathrm{~mm}$. Corola de (15)17-20 mm 3. O. mutelii

6. Labio inferior de la corola conspicuamente glandular ciliado.

6'. Labio inferior de la corola no ciliado o subglabro

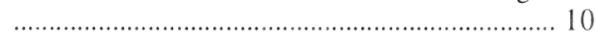

7. Segmentos del cáliz enteros (fig. 2: 7). Numerosos pelos de la corola oscuros al menos en la base o en el ápice 7. O. alba

7'. Segmentos del cáliz bífidos o bidentados, raramente enteros. Pelos de la corola incoloros, amarillos o naranjas 8

8. Filamentos insertos a (2)4-5 $\mathrm{mm}$ de la base de la corola. Corola marrón rojiza.

8. O. haenseleri

8'. Filamentos insertos a $1-2(2,5) \mathrm{mm}$ de la base de la corola. Corola marrón, marrón-amarillenta o marrón pálido con venas reticuladas, usualmente rojo oscuro en el ápice. 9

9. Hojas de lanceoladas a ovadas de 0,4-0,7 cm de anchura. Corola marrón o marrón amarillenta, rojo oscuro interiormente

15a. $O$. gracilis var. gracilis

9'. Hojas de ovadas a ovado-deltadas de $(0,6) 0,8$ $1 \mathrm{~cm}$ de anchura. Corola marrón con venas reticuladas oscuras

15b. O. gracilis var. spruneri

10. Corola rojo oscuro (a veces negruzco) exterior e interiormente, amarillenta en la base, subglabra. Filamentos insertos a 1-2 mm de la base de la corola 16. O. foetida subsp. broteri 10'. Corola no rojo oscuro exterior e interiormente, de subglabra a pubescente glandular. Filamentos insertos a más de $2 \mathrm{~mm}$ de la base de la corola 11

11. Corola galeada (fig. 2: 14), con pelos glandulares subsésiles. Filamentos insertos a $8-12 \mathrm{~mm}$ de la base de la corola

14. O. latisquama

11'. Corola no galeada, villosa glandular, pubescente glandular o subglabra. Filamentos insertos a 2$6(8) \mathrm{mm}$ de la base de la corola .................. 12

12. Corola azul oscuro (a veces negruzco) en la mitad superior, conspicuamente hinchada en la base (fig. 2: 6). Filamentos insertos cerca de la mitad de la corola.

6. O. cernua

12 '. Corola de amarillenta a ocre, no hinchada en la base. Filamentos insertos por debajo del tercio inferior de la corola. 13

13. Inflorescencia de (9)20-35 cm. Corola de 18-28 $\mathrm{mm}$ con labios grandes y marcadamente divergentes (fig. 2:9) ................ 9. O. crenata

13'. Inflorescencia de (8) 10-20 cm. Corola de 6-22 $\mathrm{mm}$ con labios pequeños, los superiores usualmente porrectos o patentes ................... 14

14. Segmentos del cáliz connatos en cerca de la mitad de su longitud (fig. 2: 11)...

11. O. almeriensis

14'. Segmentos del cáliz libres ............................. 15

15. Corola de (10)13-16 mm. Cáliz de 6-10 mm, segmentos usualmente enteros, raramente bífidos (fig. 2: 13) ........................ 13. O. minor

15'. Corola de 16-22 mm. Cáliz de 10-17 mm, segmentos desigualmente bidentados o bífidos, raramente enteros 16

16. Segmentos del cáliz de 10-13 mm bífidos (fig. 2: 10) o enteros. Corola frecuentemente genuflexa cerca de la base, ocre teñida de púrpura oscuro en la parte superior

10. O. amethystea

16'. Segmentos del cáliz de 12-17 mm bidentados (fig. 2: 12) o enteros. Corola no genuflexa, de amarillenta a ocre

12. O. artemisiae-campestris

\section{DIAGNOSIS Y CATÁLOGO FLORÍSTICO}

Sect. Trionychon Wallr.

Flores con dos bractéolas, escasamente 
pediceladas, a menudo sésiles. Cáliz con los segmentos unidos, campanulado, profunda y regularmente tetradentado con dientes dispuestos por pares lateralmente, a veces con un quinto diente de menor tamaño situado posteriormente.

1. Orobanche ramosa L., Sp. Pl. 633 (1753) Phelypaea ramosa (L.) C.A. Mey.

Ic.: Beck Von Mannagetta (1930: 62, fig. A); Bonnier (1911-1935: pl. 463, fig. 2144 [sub Phelypaea ramosa]); Rumsey \& Jury (1991: 275, fig. 1-A; 278, fig. 4-A).

Tallo de 17-31 x 0,1-0,4 cm engrosado en la base $(0,4-1,2 \mathrm{~cm})$, ramificado, pubescente glandular, blanquecino. Hojas de 3-7 mm, ovado-lanceoladas, agudas. Inflorescencia de (4)10-16 x 1,5-2,5 cm laxa, con numerosas flores; brácteas de 4,5-6 $\mathrm{mm}$ ovadolanceoladas; bractéolas de 3-4,5 mm linearlanceoladas. Cáliz de 4-6 mm, con dientes triangulares subulados o acuminados, aproximadamente iguales que el tubo. Corola de 12-15(17) mm, erecto-patente, hinchada en la base y tubular-infundibuliforme en el ápice, pubescente glandular, blanquecina o azul pálido en el ápice. Lóbulos del labio inferior elípticos, obtusos. Filamentos insertos a 3-4,5 mm de la base de la corola, glabros o subglabros; anteras glabras, blancas. Estigma blanco o violeta pálido.

Sobre plantas cultivadas en el piso termomediterráneo. 200-400 m. VI.

Material estudiado: ALMERÍA: Presa Níjar, 2-VI-62, R. Sagredo, H $^{\circ}$ LA SALLE s/n.

2. Orobanche nana (Reut.) G. Beck, Biblioth. Bot. 19:91 (1890)

O. ramosa L. subsp. nana (Reut.) Cout.
Phelypaea nana (Reut.) Rchb. fil.

Phelypaea mutelii (F.W. Schultz) Reut. var. nana Reut.

Ic.: Coste (1937: 63, fig. 2805 [sub Phelypaea nana])

Tallo de $8-12 \times(0,1) 0,15-0,3 \mathrm{~cm}$ engrosado o bulboso en la base $(0,4-0,7 \mathrm{~cm})$, simple o ramificado, pubescente glandular, blanquecino. Hojas de (3)5-9(12) mm ovadas, raramente lanceoladas. Inflorescencia de 3,5$6(8) \times(1) 2-2,7 \mathrm{~cm}$ generalmente densa en el ápice, con pocas flores; brácteas de 5-7(8) mm ovado-lanceoladas; bractéolas linearlanceoladas. Cáliz de 5-7,5 mm, con dientes triangular-acuminados de ápices filiformes, aproximadamente de la misma longitud del tubo. Corola de 12-16(17) mm de erecto-patente a patente, un tanto hinchada en la base y tubularinfundibuliforme en el ápice, pubescente glandular, blanquecina en la base, azul brillante en el ápice. Lóbulos del labio inferior elípticos más o menos agudos. Filamentos insertos a 4 $5,5 \mathrm{~mm}$ de la base de la corola, escasamente pelosos en la parte inferior; anteras glabras, blancas. Estigma blanco.

En herbazales subnitrófilos del piso termomediterráneo, sobre Aetheorhiza bulbosa. 0-1100 m. III-V.

Material estudiado: ALMERÍA: Vivero de los Alamicos, 30SWG6772, 1210 m, 24-V1988, Blanca, Cueto, Rebollar \& Robles, ALME s/n. Barranco del Caballar, sobre Galium verrucosum, s/f, $R$. Sagredo, $\mathrm{H}^{\circ}$ LA SALLE s/ n. Níjar, El Hoyazo, sobre Artemisia, s/f, $R$. Sagredo, $\mathrm{H}^{\circ}$ LA SALLE s/n. Sierra de Filabres, 1100 m, 1-V-1958, R. Sagredo, $\mathrm{H}^{\circ}$ LA SALLE s/n. Barranco Palmer, 24-III-1960, R. Sagredo, $\mathrm{H}^{\circ}$ LA SALLE s/n. Barranco Palmer, sobre Crepis bulbosa, 29-III-60, R. Sagredo, $\mathrm{H}^{\circ}$ LA SALLE $s / n$. Barranco Palmer, sobre Aetheorhiza bulbosa, 13-IV-1960, R. Sagredo, 
$\mathrm{H}^{\circ}$ LA SALLE s/n. El Romeral, 16-V-1962, $R$. Sagredo, $\mathrm{H}^{\circ}$ LA SALLE s/n. Punta del Sabinar, arenas, 15-IV-1984, B.D. Garretas \& A. Asensi, MGC 17954.

3. Orobanche mutelii F.W. Schultz in Mutel, Fl. Franç. 2:353 (1835) Cout.

O. ramosa L. subsp. mutelii (F.W. Schultz)

Phelypaea mutelii (F.W. Schultz) Reut.

Ic.: Bonnier (1911-1935: pl. 463, fig. 2144b [sub Phelypaea mutelii]); Coste (1937: 64, fig. 2806 [sub Phelypaea mutelii])

Tallo de (7)12-17(20) x 0,25-0,4(0,6) cm generalmente no engrosado en la base, simple o ramificado, pubescente glandular, blanquecino. Hojas de 6-11 mm ovadas, raramente lanceoladas. Inflorescencia de (2,5)5-8 x 2,5-3,5 cm usualmente densa, con numerosas flores; brácteas de $6-9 \mathrm{~mm}$ ovadolanceoladas; bractéolas de 6-8 mm linearlanceoladas, raramente connatas al cáliz. Cáliz de 7-10 mm, con dientes triangular-acuminados o lanceolado-subulados, aproximadamente de la misma longitud que el tubo. Corola de (15)17$20 \mathrm{~mm}$ de erecta a patente, hinchada en la base y tubular-infundibuliforme en el ápice, pubescente glandular, blanquecina en la base, de azul pálido a brillante en el ápice. Lóbulos del labio inferior redondeados obtusos, ciliados. Filamentos insertos a (4)6-7 mm de la base de la corola, escasamente pelosos en la parte inferior; anteras glabras o pelosas en la base, blancas. Estigma blanco o azul pálido.

En matorrales xerofíticos degradados de los pisos termo y mesomediterráneo, sobre Artemisia barrelieri (?), Asteriscus maritimus y Leontodon taraxacoides subsp. longirostris. 20-1500 m. III-VI.
Material estudiado: ALMERÍA: Cortijo de Gógora, sobre Sonchus y Launaea, 19-III1943, Hno. Jerónimo, ALME 1720. Al pie de Sierra Cabrera, sobre Leguminosa, 22-IV-1954, Hno. Jerónimo, ALME 1725. Venta de los Yesos (entre Tabernas y Sorbas), litosuelo de yesos del Mioceno Superior, 30SWG60, 500550 m, 7-V-1983, R. Lázaro Suau, ALME s/n. Pico María, Sierra María, 30SWG7170, 2-VII1987, Blanca \& Cueto, ALME s/n. Cerca de los Cuernos, Sierra María, 30SWG6465, 1500 m, 8-VI-1988, M. Cueto, ALME s/n. Cerca de la Ermita de la Virgen de la Cabeza, Sierra María, 30SWG7271, 20-VI-1988, M. Cueto, ALME s/ n. Arroyo del Puntal del Moral, Sierra María, 30SWG7071, 1400 m, 22-VI-1988, M. Cueto, ALME s/n. Cerro Vela Blanca, Cabo de Gata, sobre Asteriscus maritimus, 30SWF7364, 20 m, 17-III-1990, A. Pujadas et al., COA 13805. Sierra Almagro, 30SWG9936, 11-V-1990, E. Martínez, COA 17449. Playa de Los Muertos, Carboneras, 30SWF9890, 20 m, 13-IV-1994, A. Pujadas \& P.Poyato, COA 17450. Puertecico, Sta. María de Nieva, 30SWG8953, 850 m, 11-V-1994, A. Pujadas, COA 17451. Base de Sierra Cabrera, playa del Castillejo de Mocenas, entre albaidal, 30SXG0204, 5-IV1990, J. Peñas \& $M^{a}$.J. Salinas, GDAC 33375. Sierra María, sobre Thymus, s/f, R. Sagredo, $\mathrm{H}^{\circ}$ LA SALLE s/n. Venta de los Castaños, s/f, R. Sagredo, $\mathrm{H}^{\circ}$ LA SALLE $\mathrm{s} / \mathrm{n}$. Barranco Palmer, sobre Picridium, 18-III-1959, $R$. Sagredo, $\mathrm{H}^{\circ}$ LA SALLE s/n. Barranco $\mathrm{S}$. Indalecio, 6-IV-1959, R. Sagredo, $\mathrm{H}^{\circ} \mathrm{LA}$ SALLE s/n. Sierra de María, 27-VI-1962, $R$. Sagredo, $\mathrm{H}^{\circ}$ LA SALLE s/n. Barranco Palmer, sobre Leontodon taraxacoides subsp. longirostris, 13-IV-1966, R. Sagredo, $\mathrm{H}^{\circ} \mathrm{LA}$ SALLE s/n. Roquetas, 3-IV-1969, R. Sagredo, $\mathrm{H}^{\circ}$ LA SALLE s/n. Hortichuelas Bajas, 19-IV1973, R. Sagredo, H $^{\circ}$ LA SALLE s/n. Sierra Alhamilla, 30SWF49, 5-IV-1983, Lázaro Suau, MGC 13410. 

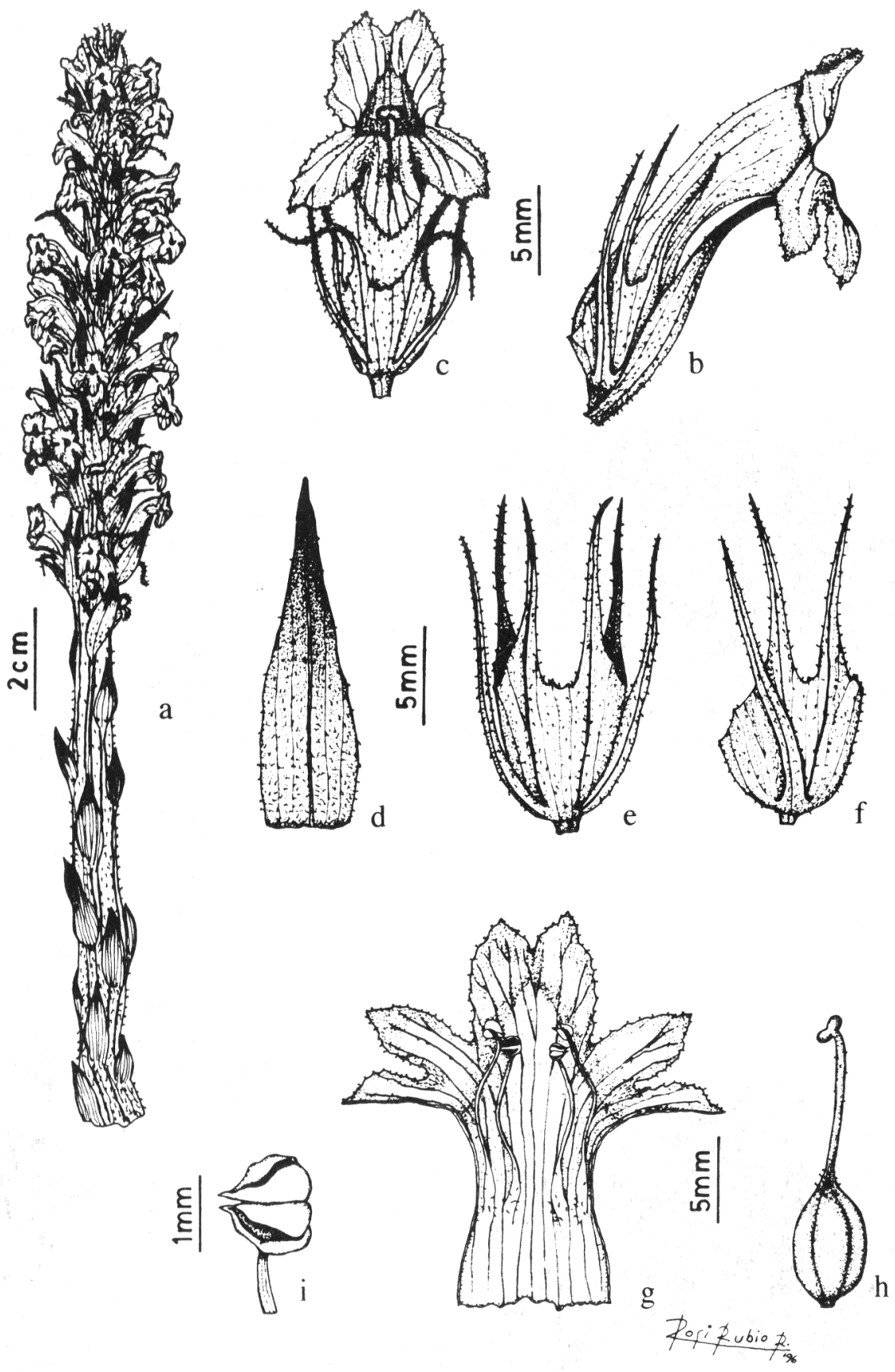

Figura 3. Orobanche schultzii: a) hábito; b) flor, visión lateral; c) flor, visión frontal; d) bráctea; e) bractéolas y cáliz, visión frontal; f) bractéola y cáliz, visión lateral; g) corola abierta y androceo; h) gineceo; i) antera. Orobanche shultzii: a) appearance; b) flower, side view; c) flower, front view; d) bract; e) bracteoles and calyx, front view; f) bracteole and calyx, side view; g) open corolla and androecium; $h$ ) gynoecium; i) anther. 
4. Orobanche schultzii Mutel, Fl. Franç. 2:429 (1835)

$$
\text { Ic.: Fig. } 3
$$

Tallo de (12)16-23 x 0,4-0,9 cm no engrosado en la base, simple, pubescente glandular, blanquecino. Hojas de (8)10-15(17) $\mathrm{mm}$ ovado-lanceoladas o lanceoladas. Inflorescencia de 6-12 x 3-3,7 cm densa, con ápice ligeramente piramidal; brácteas de 1216(20) mm lanceoladas; bractéolas de 10-13 mm linear-lanceoladas. Cáliz de (12)14-16 mm, con dientes 1,5-2 veces la longitud del tubo, linear-lanceolados con ápice filiforme. Corola de (17)19-21 mm, erecto-patente, ligeramente curvada, estrechamente campanulada, pubescente glandular, blanca con limbo teñido de azul pálido. Lóbulos del labio inferior elípticos, agudos, cortamente ciliados. Filamentos insertos a (4)6-7 mm de la base de la corola, escasamente pelosos en la parte inferior; anteras glabras, blancas. Estigma blanco.

En matorrales degradados de los pisos termo y mesomediterráneo, sobre Thapsia villosa (?). 800-1000 m. IV-V(VI).

Material estudiado: ALMERÍA: Puerto de Sta. María de Nieva, sobre Thapsia villosa 30SWG8658, 900 m, 30-V-1994, A. Pujadas, COA 17465.

\section{Orobanche laevis L., Sp. Pl. 632 (1753)}

$O$. arenaria Borkh.

Phelypaea arenaria (Borkh.) Walp.

Ic.: Beck Von Mannagetta (1930: 62, figs. B-L [sub Orobanche arenaria]); Bonnier (1911-1935: pl. 463, fig. 2142 [sub Phelypaea arenaria]); Coste (1937: 64, fig. 2809 [sub Phelypaea arenaria]); Kreutz (1995: 33 [sub Orobanche arenaria])
Tallo de 24-40(50) x 0,5-0,9 cm ligeramente o no engrosado en la base, usualmente simple, pubescente glandular, blanquecino. Hojas de (11)13-20 mm lanceoladas. Inflorescencia de 9-20 x 2-4 cm densa; brácteas de 12-15(25) mm lanceoladas; bractéolas de (9)10-15(18) linear lanceoladas. Cáliz de 13-18(21) mm, con dientes lanceoladosubulados. Corola de (18)21-30 mm de erecta a erecto-patente, campanuladoinfundibuliforme, pubescente glandular, azulviolácea, blanquecina en la base. Labio superior redondeado, obtuso, cortamente ciliado. Filamentos insertos a 3,5-6 mm de la base de la corola, subglabros o glabrescentes; anteras pelosas, blancas. Estigma blanco.

En matorrales xerofíticos degradados de los pisos meso y supramediterráneo, sobre Artemisia glutinosa. (360)900-1950 m. V-VII.

Material estudiado: ALMERÍA: Colinas hacia Filabres, s/f, s/leg., BCF 39901. Sierra entre Vélez Rubio y Sta. Ma . de Nieva, sobre Achillea millefolium, 8-VI-1951, Hno. Jerónimo, ALME 1723. Base del Maimón, 30SWG7970, $1100 \mathrm{~m}, 3-$ VI-1987, Blanca \& Cueto, ALME s/n. El Puertecico-Cruce a Los Cabrera de Abajo, talud de carretera, 30SWG8554, 850 m, 11-V-1994, A. Pujadas, COA 17330. Arroyo de El Saliente, Los Cerricos, Oria, 30SWG7254, 11-V-1994, A. Pujadas, COA 17337. Entre El Santuario y El Saliente Alto, Albox, 30SWG7453, 950 m, 11V-1994, A. Pujadas, COA 17341. Santuario El Saliente, sobre Artemisia glutinosa, 30SWG7453, 900 m, 16-V-1994, A. Pujadas, COA 17333. Calar Alto, 30SWG3819, $1950 \mathrm{~m}$, 25-IV-1994, A. Pujadas, COA 17336. Lúcar, 30SWG5141, 950 m, 25-V-1994, A. Pujadas, COA 17338. Km 3 de Cantoria a Albánchez, sobre Artemisia glutinosa, 30SWG7453, 360 m, 25-V-1994, A. Pujadas, COA 17348. Puerto de Sta. María de Nieva, 30SWG8659, 1040 m, 30-V-1994, A. Pujadas, COA 17343. Cruce de 
Los Tonosas a Vélez Rubio, sobre Artemisia glutinosa, 30SWG8663, 950 m, 30-V-1994, A. Pujadas, COA 17347. Km 1 de María a Vélez Blanco, 30SWG7474, 1100 m, 14-VI-1994, A. Pujadas, COA 17339. Aulago y cumbre de Sierra Filabres, 12-VII-1978, R. Sagredo, $\mathrm{H}^{\circ}$ LA SALLE s/n. Muela Montalbiche, 2-VII1973, R. Sagredo, $\mathrm{H}^{\circ}$ LA SALLE s/n. Alcolea, 5-VI-1959, R. Sagredo, $\mathrm{H}^{\circ}$ LA SALLE s/n. Barranco Abrucena, 30-VII-1959, R. Sagredo, $\mathrm{H}^{\circ}$ LA SALLE s/n. Camino de Vélez - De María a La Puebla - La Sagra, s/f, Clemente, MA 114831. Cañada de Clave, Vélez Rubio, sobre Artemisia, 18-V-1987, A. García Gea, MUB 40077.

\section{Sect. Orobanche}

Flores sin bractéolas, sésiles, raramente pediceladas. Cáliz generalmente partido tanto adaxial como abaxialmente, a veces con los segmentos connatos, siempre laterales y con 1 2 dientes.

6. Orobanche cernua Loefl., Iter. Hisp. 152 (1758)

Ic.: Coste (1937: 71, fig. 2825); Kreutz (1995: 77); Pignatti (1982: 610)

Tallo de 15-31 x (0,3)0,5-0,8 cm usualmente engrosado o bulboso en la base (0,9-2,0 cm), pubescente glandular, de amarillento a violeta pálido. Hojas de $8-10 \mathrm{~mm}$ ovado-lanceoladas. Inflorescencia de 4-17 x $(1,8) 2-3(3,5) \mathrm{cm}$ densa; brácteas de $8-12 \mathrm{~mm}$ ovadas. Cáliz de $7-10 \mathrm{~mm}$, de segmentos libres, enteros, bífidos o bidentados, y dientes lanceolado-subulados. Corola de 13-18 mm de erecto-patente a patente, hinchada en la base, constricta e inflexa cerca de la mitad, esparcidamente puberulenta glandular, blanquecina en la base, azul-violeta oscuro en la mitad superior. Labio superior bilobado; lóbulos del labio inferior subiguales, no ciliados. Filamentos insertos a (3)4-6(8) mm de la base de la corola, glabros o pubescentes en la parte inferior; anteras glabras o subglabras, blancas. Estigma blanco.

En matorrales xerofíticos degradados del piso termomediterráeno, sobre Artemisia barrelieri, A. glutinosa y Launaea lanifera. 20-800(1500) m. III-VI(VIII).

Material estudiado: ALMERÍA: La Hoya, 16-III-1958, Hno. M. Mauricio y R. Sagredo, ALME 1721. Serón, Las Menas, sobre Artemisia, 1300 m, 11-VIII-1958, Hno. M. Mauricio \& R. Sagredo, ALME 1718. $10 \mathrm{~km} \mathrm{E}$ Tabernas, Venta de los Yesos, growing on Artemisia in wheatfield, 30SWG60, 520-550 m, 3-VI-1967, P.W. Ball, A.O. Chater, I.K. Ferguson \& B. Valdés, ALME 1705. NW Almería, Barranco de Cerro Caguela, $\mathrm{N}$ side of Rambla de Belén, stony slopes, limestone, 30SWG47, 100-300 m, 6-VI-1967, P.W. Ball, A.O. Chater, I.K. Ferguson \& B. Valdés, ALME 1706. Entre Tabernas y Gérgal, Yesoncillo de Enmedio (c. estación de tren de Fuente Santa) en yesos del Mioceno Superior, 30SWG40, 500-600 m, 30-IV-1983, R. Lázaro Suau, ALME s/n. Cerca de los Cuernos, 30SWG6465, 1500 m, 8-VI-1988, M. Cueto, ALME s/n. C. Tabernas, sobre Artemisia barrelieri, 30SWF4898, 400 m, 29-IV-1989, A. Pujadas \& P. Poyato, COA 13632. Níjar, 30SWF7191, 17-III-1990, A. Pujadas et al., COA 13464. Salinas Cabo de Gata, 30SWF7069, 15 m, 17 III-1990, A. Pujadas et al., COA 17366. Cabo de Gata, Cerro Vela Blanca, 30SWF7364, 20 m, 17-III-1990, A. Pujadas et al., COA 17368. Los Genoveses, Cabo de Gata, 30SWF3976, 250 m, 17-III-1990, A. Pujadas et al., COA 17369. San José, 30SWF7968, 10-VII-1990, A. Pujadas, COA 17358. Yesera de Gafarillos, Sorbas, 30SWF8702. 18-IV-1992, E. Hernández \& P. Contreras, COA 17365. 
Barriada de Alcora, 30SWF2391, 760 m, 13V-1993, A. Pujadas, A. Lora \& M. Mazariegos, COA 17359. Cerro del Buho, Tabernas, sobre Artemisia barrelieri, 30SWG5202, 14-V-1993, A. Pujadas, A. Lora \& M. Mazariegos, COA 17360. Cerro Santa Fe, sobre Artemisia barrelieri, 30SWF7670, 350 m, 23-III-1994, A. Pujadas, COA 17355. Enix, sobre Artemisia barrelieri, 30SWF3879, 7-IV-1994, A. Pujadas, COA 17363. Cerro Majada Redonda, sobre Launaea lanifera, 30SWF8174, $300 \mathrm{~m}$, 13-IV-1994, A. Pujadas, COA 17353. N del Barranco del Sabinar, cortijo El Rincón de Martos, 30SWF7470, 300 m, 19-IV-1994, A. Pujadas, COA 17356. Barranco El Palmer, 30SWF3976, 250 m, 22-IV-1994, A. Pujadas, COA 17370. Cerro El Fraile, sobre Artemisia barrelieri, 30SWF8271, $40 \mathrm{~m}, 2-\mathrm{V}-1994$, A. Pujadas, COA 17371. El Garbanzal, sobre Artemisia barrelieri, 30SWF7874, 200 m, 6V-1994, A. Pujadas, COA 17352. Las Pocicas, sobre Artemisia glutinosa, 30SWG7548, 700 m, 11-V-1994, A. Pujadas, COA 17349. Arroyo del Saliente, Los Cerricos, Oria, 30SWG7254, 1000 m, 11-V-1994, A. Pujadas, COA 17372. Santuario El Saliente, sobre Artemisia glutinosa, 30SWG7453, 900 m, 16-V-1994, A. Pujadas, COA 17350. Barranco del Tartel, Sierra de Gádor, sobre Artemisia barrelieri, 500 m, 22-V-1994, A. Pujadas, COA 17351. Cerro de Mónsul, 30SWF7667, 200 m, 1-VI1994, A. Pujadas, COA 17357. El Saliente, sobre Artemisia barrelieri, 11-V-1995, A. Pujadas \& A. Lora, COA 17361. Majada Redonda, 30SWF87, 13-V-1995, A. Pujadas \& A. Lora, COA 17364. Palacio de Arboleas, 30SWF4877, 23-IV-1982, P. Sánchez, GDAC 14360. Adra, carretera a La Parra, 30SVF9469, 17-IV-1990, M.J. Martínez \& L. Gutiérrez, GDAC 35199. Las Menas, Serón, sobre Artemisia, $1300 \mathrm{~m}$, s/f, R. Sagredo, $\mathrm{H}^{\circ} \mathrm{LA}$ SALLE s/n. Km 138 entre Rioja y Tabernas, 19-IV-1967, R. Sagredo, $\mathrm{H}^{\circ}$ LA SALLE s/n. Venta de los Yesos, a $2 \mathrm{Km}$ de Uleila, 19-IV1967, R. Sagredo, $\mathrm{H}^{\circ}$ LA SALLE s/n. El Fonte, cortijo, 19-IV-1967, R. Sagredo, $\mathrm{H}^{\circ}$ LA SALLE s/n. Aguadulce, 19-IV-1968, R. Sagredo, $\mathrm{H}^{\circ}$ LA SALLE s/n. Faro Cabo de Gata, 3-V-1968, R. Sagredo, $\mathrm{H}^{\circ}$ LA SALLE s/n. Los Castaños, 14-IV-1973, R. Sagredo, $\mathrm{H}^{\circ}$ LA SALLE s/n. Serrata, 20-IV-1973, R. Sagredo, H $^{\circ}$ LA SALLE s/n. Carretera a Tabernas, Km 133 carr. general, junto a Rambla de Tabernas, 30SWG60, 450 m, 2-III-1955, P. Montserrat, JACA 5255. Carboneras, près d'Agua Amarga, lieux rocailleux incultes, $10 \mathrm{~m}, 12-\mathrm{V}-1982, \mathrm{~A}$. Charpin \& C. Defferrard, MA 294125.

7. Orobanche alba Stephan ex Willd., Sp. P1. 3:350 (1800)

O. epithymum DC.

Ic.: Beck Von Mannagetta (1930: 143, figs A-H); Bonnier (1911-1935: pl. 465, fig. 2156 [sub Orobanche epithymum]); Coste (1937: 69, fig. 2816 [sub Orobanche epithymum]); Kreutz (1995: 49); Pignatti (1982: 611); Reichenbach (1829: DCLVIII [sub Orobanche epithymum]); Rumsey \& Jury (1991: 275, fig. 1-D y 278, fig. 4-D)

Tallo de 19-27 x 0,4-0,5 cm escasamente o no engrosado en la base $(0,7 \mathrm{~cm})$, pubescente glandular, rosado-amarillento. Hojas de (10)12$20 \mathrm{~mm}$ ampliamente lanceoladas. Inflorescencia de 6-11 x 2-2,4 cm densa en la parte superior, más o menos laxa en la inferior; brácteas de 10-15 mm lanceoladas. Cáliz de 7 $10 \mathrm{~mm}$, de segmentos libres, enteros, lanceolado-subulados. Corola de 15-19 mm erecto-patente, cilíndrico-campanulada, ligeramente curvada, puberulenta glandular con muchos de los pelos rojo oscuro o púrpuras al menos en la base o en el ápice, rosadoblanquecina. Labio superior bilobado; labio inferior con lóbulos subiguales ligeramente ciliado glandulares. Filamentos insertos a 2-3 $\mathrm{mm}$ de la base de la corola, densamente 

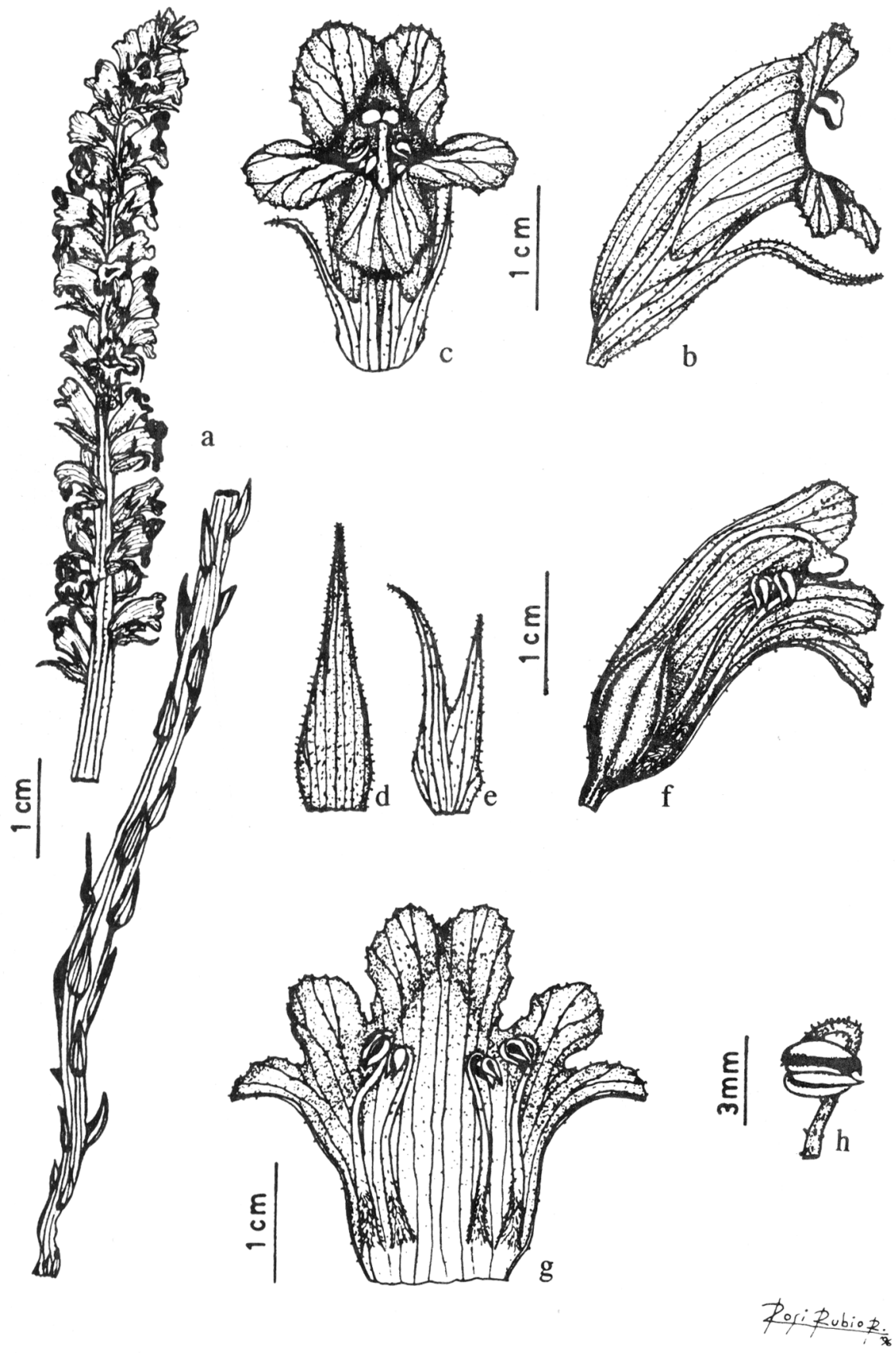

Figura 4. Orobanche haenseleri: a) hábito; b) flor, visión lateral; c) flor, visión frontal; d) bráctea; e) segmento del cáliz; f) sección longitudinal de la corola con androceo y gineceo; g) corola abierta y androceo; h) antera. Orobanche haenseleri: a) appearance; b) flower, side view; c) flower, front view: d) bract; e) segment of the calyx; f) longitudinal section of the corolla with androecium and gynoecium; $g$ ) open corolla and androecium; $h$ ) anther. 
puberulentos en la parte inferior y con pelos glandulares subsésiles en la superior; anteras subglabras, marrón pálido. Estigma púrpura.

En matorrales xerofíticos de los pisos meso y supramediterráeno, sobre tomillares. $1600 \mathrm{~m}$. VI.

Material estudiado: ALMERÍA: PeñaLobera, Sierra de Lúcar, 30SWG4745, 1600 m, 15-VI-1994, A. Pujadas \& A. Pallarés, COA 17323.

8. Orobanche haenseleri Reut. in DC., Prodr. $11: 22(1847)$

$$
\text { Ic.: Fig. } 4
$$

Tallo de (23) 40-62 x 0,6-0,8 cm usualmente engrosado en la base $(1,1-3,1 \mathrm{~cm})$, pubescente glandular, amarillento teñido de rosa. Hojas de 16-30 mm lanceoladas. Inflorescencia de 15-20 x 2,5-3,5 cm densa en la parte superior, laxa en la inferior; brácteas de 15-30 mm. Cáliz de 12-18 mm, de segmentos libres, desigualmente bidentados, raramente enteros, y con dientes lanceolado-acuminados. Corola de 20-25 mm erecto-patente, ampliamente tubular-campanulada, ligeramente curvada, densamente pelosa glandular, anaranjado-rojiza. Labio superior bilobado; labio inferior con lóbulos desiguales de margen cortamente ciliado. Filamentos insertos a (2)4-5 mm de la base de la corola, densamente pelosos en la parte inferior, glabros en la superior; anteras glabrescentes, amarillas. Estigma amarillo.

En herbazales mesofíticos del piso supramediterráeno, sobre Helleborus foetidus. 1050-2450 m. VI-VII.

Material estudiado: ALMERÍA: Cuenca alta del río Andarax, Laujar, sobre Helleborus foetidus, 30SWF1097, $1050 \mathrm{~m}, 7$-VII-1990, A.
Pujadas et al., COA 16497. Cerro Almirez, Sierra Nevada, Laujar, 30SWG0804, 2450 m, 8-VII-1990, A. Pujadas et al., COA 16496. Subida al Almirez desde Laujar, sobre Helleborusfoetidus, 30SWG0703, 2000 m, 27 VI-1994, A. Pujadas, COA 16494.

9. Orobanche crenata Forssk., Fl. Aegypt. 113 (1775)

o. speciosa DC.

Ic.: Bonnier (1911-1935: pl. 465, fig. 2155 [sub Orobanche speciosa]); Coste (1937: 69, fig. 2818 [sub Orobanche speciosa]); Reichenbach (1829: DCLXXVIII, fig. 911); Rumsey \& Jury (1991: 276, fig. 2-E y 280, fig. 6-B)

Tallo de (20)30-60 x $(0,6) 1-1,3(1,8) \mathrm{cm}$ generalmente engrosado en la base $(1,1-2,6$ $\mathrm{cm})$, villoso glandular, amarillento, marrón o púrpura. Hojas de 15-25(30) mm lanceoladas. Inflorescencia de (9)20-35 x 3,5-4,5 cm densa en la parte superior, usualmente laxa en la inferior; brácteas de 14-20 mm lanceoladas, largamente subuladas. Cáliz de 13-18 mm, de segmentos libres, profundamente bidentados, a veces enteros, y con dientes estrechamente lanceolado-subulados de ápice filiforme. Corola de 18-28 mm de erecto-patente a patente, derecha, tubular-campanulada, pubescente glandular, blanca, con limbo usualmente con venas lilas. Labios divergentes, el inferior con grandes lóbulos, el central mayor que los otros, no ciliados. Filamentos insertos a 2-3(4) mm de la base de la corola, densamente pelosos en la parte inferior, con pelos glandulares cortos en la superior; anteras glabras o subglabras, marrón grisáceas. Estigma amarillento, naranja o rosado.

En campos de cultivo del piso termomediterráeno, sobre diversas Leguminosae. 20-1500 m. IV-V. 
Material estudiado: ALMERÍA: Berja, sobre Leguminosa, 19-IV-1951, Hno. Jerónimo, ALME 1726. Cerca de la Ermita de la Virgen de la Cabeza, 30SWG7172, $1420 \mathrm{~m}$, 22-VI-1988, M. Cueto, ALME s/n. Adra, Lance de La Virgen, 30SVF9567, 10-V-1989, M.D. Fernández, COA 13003. Aguadulce, sobre Leguminosas de cultivo, IV-1957, Losa España \& Rivas Goday, MAF 89109. Venta de los Yesos, 30SWG60, 15-V-1981, Lázaro Suau, MGC 13089.

10. Orobanche amethystea Thuill., Fl. Env. Paris. ed. 2, 317 (1800)

O. castellana (Reut.) Rouy

Ic.: Beck Von Mannagetta (1930: 173, fig. 5); Bonnier (1911-1935: pl. 467, fig. 2166); Coste (1937: 73, fig. 2832)

Tallo de 20-31 x 0,4-0,8 cm escasamente o no engrosado en la base, villoso glandular, amarillento, rosado o púrpura. Hojas de (10)1420(25) $\mathrm{mm}$ lanceolado-acuminadas. Inflorescencia de 10-16 x 2-3,5 cm densa en la parte superior, laxa en la inferior; brácteas de (12)14-17 mm lanceolado-acuminadas. Cáliz de 10-13 mm, de segmentos libres, desigualmente bífidos o enteros, largamente acuminados, con ápices filiformes. Corola de 16-22 mm de erecto-patente a patente, tubular, frecuentemente inflexa cerca de la base, con dorso más o menos recto, pubescente glandular, blanco amarillenta con limbo teñido de púrpura o violeta. Labio superior bilobado; lóbulos del labio inferior subiguales, no ciliados. Filamentos insertos a (2)2,5-4(5) mm de la base de la corola, pelosos en la parte inferior, glabros o subglabros en la superior; anteras glabras, púrpuras. Estigma púrpura.

Nota: creemos que no existen razones suficientemente justificadas para separar de esta especie a $O$. castellana $(=0$. amethystea subsp. castellana) ni a nivel subespecífico.

En herbazales xerofíticos degradados de los pisos termo y mesomediterráneo, sobre Andryala ragusina y Eryngium campestre. 201800 m. IV-VI(VII).

Material estudiado: ALMERÍA: Punta Entinas, sobre Leguminosa, 8-IV-1954, Hno. Jerónimo, ALME 1722. Barranco del Caballar, 24-IV-1958, s/leg., ALME 1710. Junto al Collado del Peral, 30SWG7568, 1175 m, 27. V-1987, M. Cueto, ALME s/n. Vivero Los Alamicos, 30SWG6772, 1210 m, 24-V-1988, Blanca, Cueto, Lázaro \& Robles, ALME s/n. Cerca de la Ermita de la Virgen de la Cabeza, 30SWG7272, 1360 m, 26-V-1988, G. Blanca, M. Cueto \& R. Lázaro, ALME s/n. Cerca del Cerro de Juan López, 30SWG5164, 1500 m, 7 VI-1988, M. Cueto, ALME s/n. Subida al Cerro Almirez, Laujar, sobre Eryngium campestre, 30SWG0805, 8-VII-1990, A. Pujadas et al., COA 17327. Mojácar, 30SXG0212, 60 m, 14V-1993, A. Pujadas, A. Lora \& M. Mazariegos, COA 16461. Turre, Solonchaks, 30SWG9812, 90 m, 14-V-1993, A. Pujadas, A. Lora \& M. Mazariegos, COA 16462. Umbría de la Virgen, Sierra María, sobre Eryngium campestre, 30SWG7272, 14-VI-1994, A. Pujadas, COA 16463. N-324, Km 307, $1 \mathrm{Km}$ al $\mathrm{N}$ del Río Andarax, 30SWF3893, 300 m, 25-IV-1994, A. Pujadas, COA 16480. Santuario El Saliente, Albox, sobre Andryala ragusina, 30SWG7453, 900 m, 16-V-1994, A. Pujadas, COA 16477. Urbanización El Retamar, sobre Andryala ragusina, 30SWF6179, 30 m, 19-V-1994, A. Pujadas y R. Jiménez, COA 16476. Lúcar, sobre Andryala ragusina, 30SWG5140, 950 m, 25-V-1994, A. Pujadas, COA 16471. Km 3 Cantoria-Albánchez, sobre Andryala ragusina, 30SWG7443, 25-V-1994, A. Pujadas, COA 16474. Barranco Santa Fe de Mondújar, 30SWF4294, 250 m, 27-V-1994, A. Pujadas, COA 16470. Entre El Puertecico y Cortijo Arenales, Huércal-Overa, 30SWG8854, 850 
m, 30-V-1994, A. Pujadas, COA 16468. Bayárcal, Sierra Nevada, sobre Eryngium campestre, 30SWF0098, 7-VI-1994, A. Pallarés, COA 16466. Faro San Telmo, sobre Eryngium campestre, 30SWT4576, 20 m, 12VI-1994, A. Pujadas, COA 16465. Km 1 de María a Vélez Blanco, sobre Andryala ragusina, 30SWG7474, 1200 m, 14-VI-1994, A. Pujadas, COA 16464. Las Pocicas, sobre Andryala ragusina, 30SWG7547, 11-V-1995, A. Pujadas y A. Lora, COA 17328. Sierra de Gádor, El Sabinar, parásita de varias plantas, 1800 m, 19-VI-1992, A. Hervás F., GDAC 38288. Venta de los Yesos, $2 \mathrm{Km}$ de Uleila, 19. IV-1967, R. Sagredo, $\mathrm{H}^{\circ}$ LA SALLE s/n. Aguadulce, sobre Andryala ragusina, IV-1957, Losa España et Rivas Goday, MAF 89108. Dehesa, Chirivel, sobre Artemisia, 17-V-1987, A. García Gea, MUB 40076.

11. Orobanche almeriensis A. Pujadas, Anales Jard. Bot. Madrid 53(1) (1995)

$$
\text { Ic.: Pujadas \& Lora (1995: 51) }
$$

Tallo de 25-35 x 0,3-0,6 cm escasamente engrosado en la base, densamente villoso glandular, purpúreo. Hojas de (10)13-17 mm ovado-lanceoladas acuminadas. Inflorescencia de 12-14 x 2,5-3 cm densa; brácteas de 13-16 $\mathrm{mm}$ lanceolado-acuminadas. Cáliz de $9-12 \mathrm{~mm}$, de segmentos ampliamente connatos, desigualmente bífidos, con dientes largamente acuminados, de ápices filiformes. Corola de 17-20 mm erecto-patente, tubular, con dorso más o menos recto, densamente pubescente glandular, ocrácea con limbo teñido con venas púrpuras. Labio superior bilobado; lóbulos del labio inferior subiguales con margen subglabro. Filamentos insertos a 2-3 mm de la base de la corola, escasamente pelosos en la parte inferior, subglabros en la superior; anteras subglabras, púrpuras. Estigma púrpura.
En herbazales xerofíticos degradados del piso termomediterráeno, sobre Andryala ragusina. 900-1000 m. V.

Material estudiado: ALMERÍA: Entre El Santuario y El Saliente Alto, Sierra de las Estancias, Albox, 30SWG7453, 950 m, 2-V1994, A. Pujadas, COA 16482.

12. Orobanche artemisiae-campestris Vaucher ex Gaud., Fl. Helv. IV, 179 (1829)

O. loricata Rchb.

Ic.: Bonnier (1911-1935: pl. 467, fig. 2168); Coste (1937: 73, fig. 2833 [sub Orobanche loricata]); Kreutz (1995: 65); Reichenbach (1829: DCLXXXII, fig. 917 [sub Orobanche loricata]); Rumsey \& Jury (1991: 277, fig. 3-A y 280, fig. 6-A [sub Orobanche loricata])

Tallo de 30-35 x 0,4-0,6 cm escasamente engrosado en la base, villoso glandular, amarillento. Hojas de 15-25 mm estrechamente lanceoladas. Inflorescencia de 13-16 x 2,5-3 cm densa; brácteas de 18-25 mm estrechamente lanceoladas. Cáliz de 12-17 mm, de segmentos libres, muy largamente acuminado-filiformes, desigual y profundamente bidentados, raramente enteros. Corola de $16-18 \mathrm{~mm}$ erectopatente, tubular, con dorso más o menos recto, pubescente glandular o subglabra, amarillenta. Labio superior emarginado; lóbulos del labio inferior subiguales, no ciliados. Filamentos insertos a 2,5-3,5 mm de la base de la corola, pelosos en la parte inferior, con pelos glandulares subsésiles en la superior; anteras subglabras, marrón pálido. Estigma púrpura.

En herbazales mesofíticos del piso termomediterráneo. 800-900 m. IV-V.

Material estudiado: ALMERÍA: Cóbdar, 
base del cerro Pinar, cuneta de la carretera, 30SWG7124, 800 m, 25-V-1994, A. Pujadas, COA 16475.

13. Orobanche minor Sm. in Sowerby, Engl. Bot. 6: t.422 (1797)

Ic.: Beck Von Mannagetta (1930: 173, figs. A-G); Coste (1937: 74, fig. 2835); Kreutz (1995: 121); Reichenbach (1829: DCLII, figs. 876 y 877); Rumsey \& Jury (1991: 277, fig. 3B y 280 , fig. 6-C)

Tallo de (15)20-50 x 0,3-0,7 cm ligeramente o no engrosado en la base $(0,7-1,5$ $\mathrm{cm})$, villoso glandular, blanquecino o teñido de violeta. Hojas de 10-20 mm lanceoladas acuminadas. Inflorescencia de (8)12-20 x 2$2,5 \mathrm{~cm}$ densa en la parte superior, laxa en la inferior; brácteas de 13-15(18) mm, lanceoladoacuminadas. Cáliz de $6-10 \mathrm{~mm}$, de segmentos libres usualmente enteros, a veces desigualmente bífidos, lanceoladoacuminados. Corola de (10)13-16 mm de erecto-patente a patente, tubular, con dorso ligeramente curvado, pubescente glandular, blanquecina. Labio superior cortamente emarginado; labio inferior con el lóbulo central más grande, no ciliado. Filamentos insertos a 2-3 mm de la base de la corola, pelosos en la parte inferior; anteras escasamente pelosas en la base, púrpuras. Estigma blanco.

Nota: Las plantas recolectadas en Almería corresponden a la forma albens G. Beck, Corinthia 2:108-109, Jahrgang 20 (1921).

En matorrales xerofíticos degradados del piso termomediterráeno, sobre Ballota hirsuta. 20-500(1500) m. III-V(VI).

Material estudiado: ALMERÍA: Barranco del Palmer, 22-IV-1966, s/leg., ALME 1702. $10 \mathrm{Km}$ al E de Tabernas, Venta de la Yesos, gypsum hills, 30SWG60, 520-550 m, 3-VI1967, P.W. Ball, A.O. Chater, I.K. Ferguson \& B. Valdés, ALME 1709. Venta de los Yesos entre Tabernas y Sorbas, N-340 Km 155 , litosuelo de yesos del Mioceno Superior, 30SWG60, 500-550 m, 13-V-1981, R. Lázaro Suau, ALME s/n. Barranco Mula, 30SWG6265, 1500 m, 8-VI-1988, M. Cueto, ALME s/n. Cerro Majada Redonda, sobre Ballota hirsuta, 30SWF8176, 400 m, 13-IV-1994, A. Pujadas, COA 17444. Cortijo El Rincón de Martos, sobre Ballota hirsuta, 30SWF7470, 350 m, 19 . IV-1994, A. Pujadas \& R. Jiménez, COA 17447. Cerro de Los Frailes, sobre Ballota hirsuta, 30SWF8171, $300 \mathrm{~m}, 2-\mathrm{V}-1994$, A. Pujadas, COA 17446. Agua Amarga, sobre Ballota hirsuta, 30SWF9588, $20 \mathrm{~m}, 6-\mathrm{V}-1994$, A. Pujadas, COA 17427. El Garbanzal, sobre Ballota hirsuta, 30SWF8176, 200 m, 6-V-1994, A. Pujadas, COA 17445. Barranco Palmer, sobre raíz de Ficus carica, 29-III-1960, $R$. Sagredo, $\mathrm{H}^{\circ}$ LA SALLE $\mathrm{s} / \mathrm{n}$. Alcolea, sobre Ballota, 6-V-1960, R. Sagredo, $\mathrm{H}^{\circ}$ LA SALLE s/n. Barranco del Sabinal, 16-IV-1962, $R$. Sagredo, $\mathrm{H}^{\circ}$ LA SALLE $\mathrm{s} / \mathrm{n}$. Barranco del Sabinal, 200-350 m, 2-VI-1967, R. Sagredo, $H^{\circ}$ LA SALLE s/n. Garbanzal, 16-III-1973, R. Sagredo, $\mathrm{H}^{\circ}$ LA SALLE s/n. Garbanzal, $16-$ IV-1973, R. Sagredo, $\mathrm{H}^{\circ}$ LA SALLE s/n.

14. Orobanche latisquama (F.W. Schultz) Batt. in Batt. \& Trabut, Fl. Algér., Dicot. 659 (1890)

\section{Ceratocalyx macrolepis Coss.}

Ic.: Cadevall y Font Quer (1932: 297 [sub Ceratocalyx macrolepis]; Valdés et al. (1986: vol. 2, 556)

Tallo de 30-42 x (0,4)0,5-0,8 cm no engrosado en la base, glabro, ocre o purpúreo. Hojas de 15-20 mm densas, largamente ovadooblongas. Inflorescencia de (7)11-27 x 3-4 cm densa; brácteas de 20-25 mm, deltadas. Cáliz 
de 15-20 mm, de segmentos connatos en el cuarto inferior, enteros, lanceoladoacuminados. Corola de 25-30 mm suberecta, galeada, a veces hinchada en la base, constricta hacia la mitad, campanulada en la parte superior, subglabra con pelos glandulares subsésiles, blanco-amarillenta teñida de púrpura hacia el ápice. Labio superior subentero; labio inferior con lóbulos iguales, no ciliado. Filamentos insertos a 8-12 mm de la base de la corola; anteras villosas, púrpuras. Estigma blanco.

En matorrales xerofíticos del piso termomediterráneo, sobre Rosmarinus eriocalyx y Rosmarinus officinalis. 200-1320 m. III-V.

Material estudiado: ALMERÍA: Vereda Alta, 30SWG6871, 1320 m, 26-V-1988, G. Blanca \& M. Cueto, ALME s/n. Por Cóbdar, sobre romero, s/f, Hno. Rufino, BCF 39878. Km 10 Carboneras-Níjar, sobre Rosmarinus eriocalyx, 30SWF9092, 200 m, 13-III-1994, A. Pujadas \& P. Poyato, COA 17441. Cóbdar, sobre romero, 7-III-1959, $R$. Sagredo, $\mathrm{H}^{\circ}$ LA SALLE s/n. Laujar, 7-III-1966, R. Sagredo, $\mathrm{H}^{\circ}$ LA SALLE $s / n$.

15. Orobanche gracilis Sm., Trans. Linn. Soc. London 4:172 (1798)

o. cruenta Bertol.

IC.: Bonnier (1911-1935: pl. 465, fig. 2151 [sub Orobanche cruenta]); Coste (1937: 68, fig. 2812); Kreutz (1995: 101)

Tallo de (11)25-40 x 0,5-1 cm algo engrosado o bulboso en la base $(0,8-1,2 \mathrm{~cm})$, pubescente glandular, amarillento, marrón o rojo-purpúreo. Hojas de 10-25 mm de ovadodeltadas a lanceoladas. Inflorescencia de (5)7$20 \times 2-3 \mathrm{~cm}$ usualmente densa, raramente laxa en la parte inferior; brácteas de (11)13-20 mm lanceolado-acuminadas. Cáliz de 1-1,8 mm, de segmentos libres, desigualmente bífidos 0 bidentados raramente enteros, con dientes ampliamente triangular-lanceolados, subulados. Corola de (16)18-24 mm de erectopatente a patente, de tubular a campanulada, de ligeramente curvada a recta, pubescente glandular, de amarilla a ocrácea exteriormente, a menudo con venas marrones o rojas, y rojo oscuro brillante interiormente. Labio superior bilobado, emarginado; lóbulos del labio inferior subiguales o desiguales con el lóbulo central de doble tamaño que los otros, ciliados. Filamentos insertos a 1-2(2,5) mm de la base de la corola, pelosos al menos en la parte inferior; anteras glabras o subglabras, blanquecinas. Estigma amarillo.

a) var. gracilis: hojas usualmente de 0,4-0,7 $\mathrm{cm}$ de anchura, de lanceoladas a ovadas. Cáliz sin venas conspicuas. Corola amarilla u ocrácea, con ápice exteriormente púrpura 0 rojo, rojo oscuro brillante interiormente. Filamentos pelosos en la parte inferior y pubescente, glandulares en la superior.

Nota: la forma orgeia G. Beck, Monogr. Orob., t. III, 198 (1890), con el lóbulo central del labio inferior de la corola de doble tamaño que los otros, procedente de Sierra María, ha sido frecuentemente identificado erróneamente como O. variegata Wallr.

En matorrales xerofíticos de los pisos meso y supramediterráneo, sobre Anthyllis cytisoides, Coronilla juncea, Erinacea anthyllis, Genista baetica y Ulex parviflorus. (300)1400-2400 m. (IV)V-VII.

b) var. spruneri (F.W. Schultz) G. Beck, Monogr. Orob., t. III, 198 (1890): hojas usualmente de $(0,6) 0,8-1 \mathrm{~cm}$ de anchura, de ovadas a ovado-deltadas. Cáliz con venas conspicuas (incluso en los especímenes secos). Corola ocrácea con venas reticuladas rojas o 

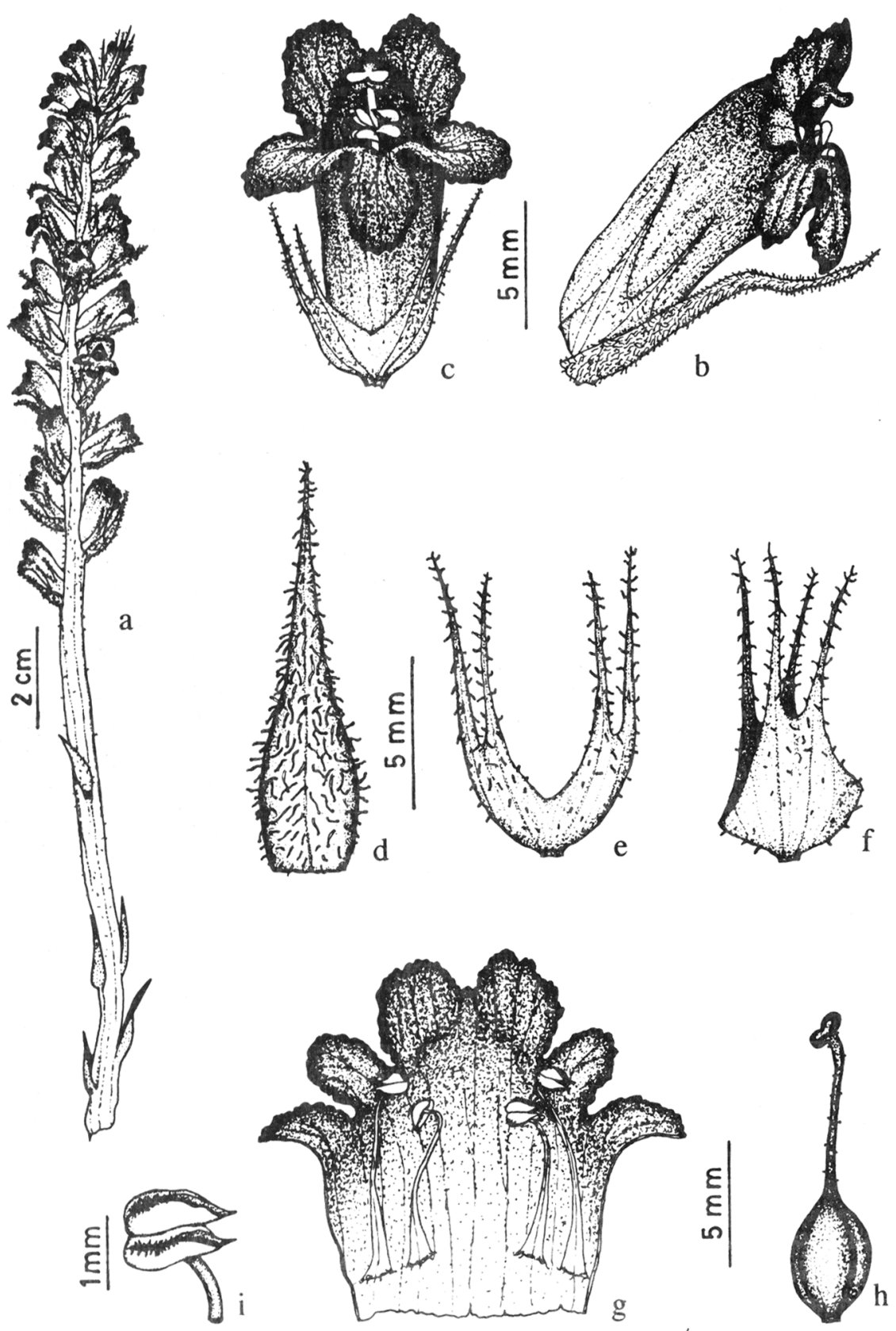

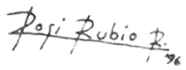

Figura 5. Orobanche foetida subsp. lusitanica: a) hábito; b) flor, visión lateral; c) flor, visión frontal; d) bráctea; e) cáliz, visión frontal; f) cáliz, visión lateral; g) corola abierta y androceo; h) gineceo; i) antera. Orobanche foetida subsp. lusitanica: a) appearance; b) flower, side view; c) flower, front view: d) bract; e) calyx, front view; g) open corolla and androecium; h) gynoecium; i) anther. 
marrones exteriormente, rojiza o no interiormente. Filamentos pelosos 0 glabrescentes en la parte inferior y subglabros en la superior.

Nota: la var. spruneri ha sido denominada a menudo $\boldsymbol{O}$. reticulata, auct. non Wallr.

En matorrales xerofíticos del piso termomediterráneo, sobre Anthyllis cytisoides y Ulex parviflorus. 250-1600 m; (II)III-V(VI).

\section{Material estudiado:}

a) var. gracilis

ALMERÍA: Sierra de Cabo de Gata, Barranco del Sabinar, $\mathrm{N}$ facing side of Monte de la Revancha, limestone slopes, 30SWF76, 2-VI-1967, P.W. Ball, A.O. Chater, I.K. Ferguson \& B. Valdés, ALME 1707. Portachico de Sierra María, 30SWG7270, 1800 m, 21-VI1988, M. Cueto, ALME s/n. Falda N Sierra María, 30SWG7472, 1460 m, 23-VI-1988, M. Cueto, ALME s/n. Cerro Majada Redonda, sobre Anthyllis cytisoides, 30SWF8176, 300 m, 13-IV-1994, A. Pujadas, COA 17377. Base de El Almirez, Laujar, sobre Genista baetica, 30SWG0804, 2400 m, 9-VI-1994, A. Pujadas, COA 17385. Calizas de la Tetica de Bacares, sobre Erinacea anthyllis, 30SWG5223, 2000 m, 10-VI-1994, A. Pallarés, COA 17379. Collado La Burrica, Sierra María, sobre Erinacea anthyllis, 30SWG7270, 1700 m, 14VI-1994, A. Pujadas, COA 17376. El Almirez, sobre Genista baetica, 30SWG0804, $2450 \mathrm{~m}$, 27-VI-1994, A. Pujadas, COA 17384. Subida al cerro Revancha, 12-V-1995, A. Lora \& A. Pujadas, COA 17378. Castala, sobre Coronilla juncea, 12-V-1960, R. Sagredo, H LA SALLE $\mathrm{s} / \mathrm{n}$. Castala, sobre Ulex parviflorus, 12-V1960, R. Sagredo, $H^{\circ}$ LA SALLE s/n. Sierra María, 2-VII-1965, s/leg., Hº LA SALLE s/n. Sierra de Bédar, 10-IV-1969, R. Sagredo, H $^{\circ}$ LA SALLE s/n. Sierra María, 2-VII-1969, $R$. Sagredo, $\mathrm{H}^{\circ}$ LA SALLE s/n. Barranco Agua del Medio, 16-IV-1974, R. Sagredo, $\mathrm{H}^{\circ}$ LA SALLE s/n.

\section{b) var. spruneri}

ALMERÍA: Rambla de La Alcazaba, Adra, 31-III-1989, M.D. Fernández, COA 13875. Estribaciones de la Sierra de Gádor, 15-V1990, B. Luque, COA 17416. Del Llano de Balsa Nueva a Castala, sobre Ulex parviflorus, 30SWF0985, 1500 m, 16-VI-1991, A. Pujadas et al., COA 17418. Fuente de la Parra, Sierra de Gádor, sobre Ulex parviflorus, 30SWF1389, 1600 m, 17-VI-1992, A. Pujadas et al., COA 17417. Barranco del Sabinar, sobre Ulex parviflorus, 30SWF7468, 7-II-1994, A. Pujadas, COA 17412. Cerro Santa Fe, mina Sta. Bárbara, 30SWF7670, 400 m, 23-III-1994, A. Pujadas, COA 17420. Cerro Majada Redonda, sobre Ulex parviflorus, 30SWF8176, 350 m, 13-IV-1994, A. Pujadas, COA 17419. Rellana, sobre Ulex parviflorus, 30SWF8277, 400 m, 16-IV-1994, A. Pujadas, COA 17415. Cortijo El Rincón de Martos, 30SWF7470, 300 m, 19-IV-1994, A. Pujadas \& R. Jiménez, COA 17410. El Garbanzal, 30SWF7874, 250 m, 6V-1994, A. Pujadas, COA 17413. Félix, sobre Anthyllis cytisoides, 30SWF3181, 9-V-1994, A. Pujadas y A. Pallarés, COA 17406. Majada Redonda, sobre Ulex parviflorus, 30SWF87, 12-V-1994, A. Lora \& A. Pujadas, COA 17408. Puerto de la Virgen, Benizalón, sobre Ulex parviflorus, 30SWG7020, 1000 m, 25-V-1994, A. Pujadas, COA 17414. Rodalquilar, sobre Ulex parviflorus, 30SWF8579, 9-IV-1995, A. Pujadas, COA 17407. Sierra de Gádor, La Parra de Fondón, parásita de varios hospedantes, $1400 \mathrm{~m}, 14-\mathrm{VI}-1992$, A. Hervás F., GDAC 38287.

16. Orobanche foetida Poir. subsp. broteri Guimarães, Broteria 3:105 (1903) o. foetida Poir. var. Iusitanica Cout.

Ic.: Fig. 5 
Tallo de 20-40 x $(0,2) 0,3-0,6(0,7) \mathrm{cm}$ ligeramente engrosado en la base $(0,7-1,4 \mathrm{~cm})$, pubescente glandular, rojizo. Hojas de 10-16 $\mathrm{mm}$ estrechamente lanceoladas. Inflorescencia de 7-13(23) x 2,4-2,6 cm densa en la parte superior, laxa en la inferior; brácteas de 10-15 mm, lanceolado-acuminadas. Cáliz de 8-11 mm, de segmentos connatos en la base, bidentados, largamente acuminados con ápices subfiliformes. Corola de (11)13-15 mm erectopatente, tubular, más o menos recta, subglabra, púrpura-rojizo oscuro, amarillenta en la base. Labio superior bilobado con lóbulos erectos; labio inferior no ciliado. Filamentos insertos a 1-2 mm de la base de la corola, glabros 0 subglabros; anteras escasamente pelosas en la sutura, amarillentas. Estigma amarillo.

Nota: frecuentemente confundido con $O$. sanguinea $\mathrm{C}$. Presl al tener también los filamentos insertos a 1-2 mm de la base de la corola.

En herbazales mesofíticos del piso mesomediterráneo, sobre Trifolium repens (?). $850 \mathrm{~m}$. VIII.

Material estudiado: ALMERÍA: Sierra de Gádor, Alcolea, parásita de diversas Leguminosas, 850 m, 7-VIII-1992, A. Hervás F., GDAC 38289.

\section{CONCLUSIONES}

Destacamos la importancia y diversidad florística de la provincia con la presencia de 16 especies de Orobanche, sobre todo si se las compara con las 22 existentes en Andalucía (Pujadas et al., 1994), las 31 en la Península Ibérica (Chater \& Webb, 1972), las 14 en las Islas Británicas (Rumsey \& Jury, 1991), las 30 en Europa Central (Kreutz, 1995) o los 48 taxones citados para toda Europa (Chater \& Webb, 1972).
El hecho de ser un géneŕo escasamente estudiado y pobremente representado en los herbarios (por ser poco recolectado por los botánicos) nos permiten suponer que la relación de especies de este estudio no sea definitiva y que puedan efectuarse nuevas adiciones al mismo.

AGRADECIMIENTOS. Al Dr. A. Pallarés por su aportación al conocimiento de estas especies. A los conservadores de los Herbarios: ALME, BC, BCC, BCF, COLEGIO LA SALLE DE ALMERÍA, GDA, GDAC, JACA, JAEN, MA, MAF, MGC, MUB, SEV por las facilidades en la consulta de sus respectivos herbarios.

\section{BIBLIOGRAFÍA}

BECK VON MANNAGETTA, G. -1890Monographie der Gattung Orobanche. Bibliotheca Botanica, 19: 1-275

BECK VON MANNAGETTA, G. -1930Orobanchaceae, in: Engler (Ed.), Das Pflanzanreich, 96 (IV-261), pp. 1-348. Berlin.

BONNIER, G. -1911/1935-Flore Complete Illustrée en couleurs de France, Suisse et Belgique. Tome VIII, fasc. 78, Paris.

CADEVALL, J. y P. FONTQUER -1932- Flora de Catalunya. Vol. IV. Barcelona.

CASTRO, H. y S. CAÑADAS - 1982- Bibliografía botánica sobre el sector corológico almeriense, in: Homenaje Almeriense al Botánico Rufino Sagredo, 171-184. Almería.

CHATER, A. O. \& D.A. WEBB -1972- Orobanche, in: Tutin et al. (Eds.), Flora Europaea, 3:286293. Cambridge.

COSTE, H. -1937-Flore descriptive et illustrée de la France de la Corse et des contrés limitrophes. Vol. III. París.

FERNÁNDEZ, C., M.C. CRUZ, M. LÓPEZPULIDO, C. AMEZCUA y D. CASADO - 1991 Flora de Andalucía. Catálogo bibliográfico de las plantas vasculares. Jaén.

GREUTER, W.R., H.M. BURDET \& G. LONG 1989- Med-Checklist. Vol.4 (LauraceaeRhamnaceae). Genève.

GUIMARAES, J.A. -1903- Monografia das Orobanchaceas. Broteria, 1-188. 
KREUTZ, C.A.J. -1995-Orobanche. The European broomrape species. Central and Northern Europe. Maastricht.

PIGNATTI, S. -1982- Flora d'Italia, Vol II. Bologna.

PUJADAS, A. y A. LORA -1995-Orobanche almeriensis A. Pujadas (Orobanchaceae), nueva especie del sureste de la Península Ibérica. Anales Jard. Bot. Madrid, 53(1): 47-53.

PUJADAS-SALVA, A., E. HERNÁNDEZBERMEJO y J.A.R. OLIVEIRA-VELLOSO 1994- The Genus Orobanche in Andalusia (southern Spain): Taxonomical, chorological \& ecological aspects, in: Pieterse, Verklej \& Ter Borg (Eds.), Biology and management of Orobanche, 132-137. Amsterdam.

REICHENBACH, H.G.L. -1829- Iconografía botanica seu Plantae critica. Icones Plantarum rariorum et minus rite cognitarum. Vol. VII. Lipsiae.

RUMSEY, F.J. \& S.L. JURY -1991-. An account of Orobanche L. in Britain and Ireland. Watsonia, 18: 257-295.

SAGREDO, R. -1987- Flora de Almería. Almería. TUTIN, T.G., V.H. HEYWOOD, N.A. BURGES, D.M. MOORE, D.H. VALENTINE, S.M. WALTERS \& D.A. WEBB (Eds.) -1964/1980Flora Europaea, Vols. 1-5. Cambridge.
VALDÉS, B., S. TALAVERA y E. FERNANDEZGALIANO (Eds). -1986- Flora Vascular de Andalucía Occidental, Vols. 1-3. Barcelona.

WILLKOMM, H.M. -1870- Orobanchaceae, in: Willkomm \& Lange (Eds.), Prodromus Florae Hispanicae 2:620-632. Stuttgart.

WILLKOMM, H.M. -1893-Supplementum prodromi florae hispanicae. Stuttgart.

Aceptado para su publicación en Mayo de 1996

Dirección de los autores. Jardín Botánico de Córdoba. Avda. de Linneo, s/n. 14004 Córdoba. 